\title{
Electrocardiograms Associated with Sudden Cardiac Death
}

\author{
An Expert Interview with Martin Borggrefe ${ }^{1,2}$
}

1. First Department of Medicine, University Medical Centre, Mannheim (UMM), Faculty of Medicine Mannheim, University of Heidelberg, Mannheim, Germany; 2. German Centre for Cardiovascular Research (DZHK) Partner Site Heidelberg/Mannheim, Mannheim, Germany

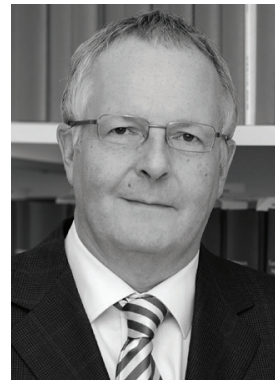

\section{Martin Borggrefe}

Professor Martin Borggrefe is the Director of Cardiology at the University Medical Centre Mannheim at the University of Heidelberg. He has a special interest in cardiac arrhythmias - from the electrocardiogram to basic science. Over the last decade, Professor Borggrefe has developed an interest in so-called 'channelopathies', which are primary electrical diseases of the heart and affect structurally normal and functioning hearts. In this field, he has discovered several novel entities, such as the short QT syndrome along with other I point elevation diseases which usually affect young people who are otherwise healthy. Professor Borggrefe was among the first physicians who developed radiofrequency catheter ablation, publishing the first paper describing ablation of a patient with Wolff-Parkinson-White syndrome using radiofrequency in 1986.

\section{Keywords}

ECG diagnosis, Sudden Cardiac Death, SCD, SCD risk

Disclosures: Martin Borggrefe has nothing to declare in relation to this article.

Acknowledgements: Editorial assistance was provided by Colin Griffin, Freelance Medical Writer and supported by Touch Medical Media.

Review Process: This is an expert interview and as such, has not undergone the journal's standard peer review process.

Authorship: The named authors meet the International Committee of Medical Journal Editors (ICMJE) criteria for authorship of this manuscript take responsibility for the integrity of the work as a whole, and have given final approval for the version to be published.

Received: 14 August 2018

Published Online: 25 October 2018

Citation: European Arrhythmia \& Electrophysiology. 2019;5(1):13-5

Corresponding Author: Martin Borggrefe, I. Medizinische Klinik, Universitätsmedizin Mannheim, Theodor-Kutzer-Ufer 1-3, 68167 Mannheim, Germany E: martin.borggrefe@umm.de

Support: No funding was received in

the publication of this article. udden cardiac death arises from the unexpected stopping of the heart and is associated with up to a half of cardiovascular deaths. We discuss with Professor Martin Borggrefe how to identify patient populations at risk of sudden cardiac death (SCD) and how we can use diagnostic tools in at-risk patients. Effective detection, diagnosis, intervention and management in at-risk patients requires a team approach to optimise preventative strategies and ultimately reduce the incidence of SCD.

\section{Q. What is known about the epidemiology of sudden cardiac death? Is it more common in some age groups or racial groups, are there any known risk factors such as family history or comorbidities?}

In Germany, approximately 120-140,000 people die suddenly each year. ${ }^{1}$ This is a substantial number - more patients die of SCD than from cancer or other diseases. It is a major epidemiological problem. Approximately 7,000 people die from channelopathies (5-8\% of those being patients with SCD), so these are deaths in patients with structurally normal hearts. The major causes of SCD are myocardial infarction or acute ischaemic event complicated by ventricular fibrillation. In the majority of people (around $80 \%$ ) who die suddenly, however, there is a prior history of myocardial infarction, and a mean of 6.5 years after myocardial infarction, the patient develops life-threatening ventricular arrhythmias. ${ }^{2}$ The other $20 \%$ of people who are affected suffer from cardiomyopathies, such as dilative cardiomyopathy or hypertrophic cardiomyopathy. However, the risk of dying suddenly in these diseases is much lower than previously published. ${ }^{3}$ So, usually, cardiomyopathies carry a benign cause, and only a minority suffer from SCD.

Looking at gender, males are more often affected than females. The reasons for this are not well understood. Another example of this is channelopathies such as Brugada syndrome, where a predominance of males are at higher risk compared to females. With regard to long QT syndrome, the gender aspect is less relevant - both sexes are affected equally. However, in the general population females have longer QT intervals and are more often affected by drug-induced QT prolongation which carries the risk of ventricular tachyarrhythmias.

With regard to racial differences, this is not well-studied. We do know that black athletes more often suffer from hypertrophy. Hypertrophy, by itself, is a risk factor for SCD. When we look into black athletes, the differential diagnosis between the athletes' heart and hypertrophic cardiomyopathy becomes quite complex. In addition, we know today that hypertrophy in black people is associated with an increased risk of SCD. 
We should also take into account that past experience suggests that athletes are often using drugs for increasing muscle mass or other drugs to enhance athletic activity. ${ }^{4}$ These drugs are interacting with muscle and the heart muscle and, thereby, might increase the propensity to develop arrhythmias in certain situations.

When we look into risk factors for SCD, it is generally observed that the more heart disease you have, the higher the risk becomes. For example, patients with prior myocardial infarction, where the extent of scarring is greater, are at higher risk of dying suddenly. When we look into hypertrophy, the thicker the muscle, the more fibrosis, the higher the risk of dying suddenly. When we look into channelopathies, the longer the QT interval, the higher the risk of dying suddenly. The shorter the corrected QT, the higher the risk to the patient. ${ }^{5}$ Thus, the extent of disease is predictive of SCD.

When we look into other risk factors, it is clear that a family history of myocardial infarction complicated by ventricular fibrillation, especially under the age of 65 , is predictive of SCD in the next generation. From the Paris study, it is evident that the children of patients who have ventricular fibrillation and acute infarctions will have a higher risk of developing this kind of complication - around a twofold increase. ${ }^{6}$

The act of taking a family history is very important in all aspects of cardiology; for example, recording details of those with Brugada syndrome or hypertrophic cardiomyopathy is important because family history of SCD - especially under the age of 40 - indicates a high risk.

\section{Q. What key echocardiogram characteristics are indicative of an increased risk of SCD and can these features be readily detected? What pathological changes do these ECGs indicate?}

Today, there is a lack of understanding of electrocardiograms (ECGS). It is not well taught during medical school, and physicians today are lacking knowledge on the basics of ECG interpretation. Therefore, I think, the ECG is underused to predict SCD.

Currently, we can detect abnormalities predictive of SCD in the ECG. The early study of preexcitation when the PR interval is short and there is a delta wave present may be indicative of a person who is otherwise healthy, but may be affected by life-threatening arrhythmias (Wolff-Parkinson-White syndrome). With regard to other abnormalities, a paper by Aro and colleagues looked into the ECG to predict SCD and found that patients with at least four ECG abnormalities had a significantly greater risk of SCD. ${ }^{7}$ When these parameters were assessed in light of the Atherosclerosis Risk in Communities study, they were highly indicative of a risk of dying suddenly in the general community. ${ }^{8}$ Beyond that, we have markers of abnormal repolarisation, which are indicative of an increased risk of SCD. When you look at the QT interval, if it is shorter than 320 milliseconds, then there is a suspicion of a short QT syndrome, which is a serious condition. People can be affected by atrial fibrillation as well as ventricular fibrillation at a young age. ${ }^{\text {To }}$ date, there has been only one report on about 80 patients with short QT syndrome, which showed a very high incidence of SCD in these families. ${ }^{10}$ When we look at the QT interval in patients with long QT syndrome, we not only assess the duration of the QT interval - the longer, the higher the risk - but also whether the angle of repolarisation is symmetrical or asymmetrical, which also an aspect that is indicative of risk.
With regard to I point elevation, in the past 30 years we have identified the so-called Brugada syndrome, which is characterised by a J point elevation in the right precordial leads. ${ }^{11}$ Some patients show a presence of conduction disease and deep $S$ waves in lead three. These ECG markers, particularly the extent of I point elevation and the presence of conduction disease, are highly indicative of a very high-risk group within patients with Brugada syndrome.

Physicians also should look at the voltage of the QS complex. For example, with low voltage and the presence of conduction disease, one might be suspicious of patients with cardiomyopathy who carry a lamin mutation, because these patients are at very high risk of dying suddenly. Another aspect of the ECG as a marker of risk, is that in some patients you can see fragmentation of the QS complex, and fragmentation means conduction disease. This is also indicative of an increased risk of SCD. When we look into the ECG in patients with hypertrophic cardiomyopathy, we can have markers that are also indicative of an increased risk with the extent of hypertrophy. The extent of voltage and repolarisation abnormalities, prolonged QT and presence of Q waves may indicate a more severe process of hypertrophy.

ECG can also be used to diagnose arrhythmogenic right ventricular cardiomyopathy, which is characterised by ST depression in leads V1 to V3 and, in some patients, low voltage and conduction disease with regard to prolonged PR interval. Sometimes this reveals fractionation at the terminal portion of the QS complex, especially in leads V1 and V2 - a so-called Epsilon potential, indicative of right parietal conduction slowing, which can not only be seen by signal averaging the ECG but also on the normally filtered ECG.

In the past 10 years, there has been interest in ventricular fibrillation associated with early repolarisation. This means that in patients with so-called idiopathic ventricular fibrillation, about one-third of them have ECC stigma of early repolarisation. ${ }^{12}$ It is J point elevation in the inferior leads, and this is indicative of potential risk of SCD. ${ }^{12}$ Early repolarisation is often present in patients with hypertrophy and in athletes, so the sensitivity of this ECG marker is quite complex.

\section{Q. Does it take an expert to identify ECG features indicative of elevated SCD risk or could a general physician, a nurse or even an automated system find them at a routine examination?}

With regard to cardiac repolarisation, I think it needs an expert. As an example, when Brugada described his first patients with Brugada syndrome, I discovered a patient with this condition 30 years ago at our hospital. I took the ECG and sent it to 500 German cardiologists; they had this photo on their desk, and from then on, they were identifying patients with this disease. You only can detect what you are aware of. Therefore, I think ECG tracings need to be reviewed by experts. I often receive phone calls or faxes and emails containing ECGs where I decide, sometimes with a small team, whether this is a disease or whether this is a person who might be at risk of SCD.

I think it is a team approach, and I don't think that a nurse or an automatic ECG machine, at present, will identify these people. But with big data and machine learning, I think in the future the ECGs will be read automatically. With higher quality data, we will improve the detection algorithms. With the current detection 
algorithms, we only can see measurements in the time domain. So, whether the intervals are short or long, this is a given that it is already possible to automatically detect. When it comes to other abnormalities, I think we will see in the future better automation and better ECG algorithms, which will lead to improvement of detection.

\section{Q. Given the extent of investigation into ECGs indicative of increased SCD risk, why is it not used on a greater scale? Is screening young (or older) populations and categorising them for increased risk possible or justifiable?}

ECG is under-used for risk prediction in the general population, but also for the patients who are affected by heart disease. In Italy, the ECG was introduced into the pre-participation screening of professional athletes and is a mandatory requirement before they are allowed to compete. Domenico Corrado has attributed this pre-participation to a dramatic decrease in the risk of SCD in Italian athletes in the last 20 years. ${ }^{13}$ Therefore, screening by ECG is helpful in reducing the risk of death.

In general, there is no screening process established in Europe. For example, there is no attempt made to screen newborns by ECG. I think it mainly has to do with the fact that ECGs can be difficult to perform in newborns as they often move too much. But I think ECGs could be developed which are more easily handheld, for example taken just over the chest of a person. We are more interested in intervals and ST segment, which can be detected by a precordial ECG.

\section{Q. What preventive measures can be taken in individuals found to have ECGs consistent with increased SCD risk?}

Take patients with a long QT syndrome for example, patients who are at increased risk and have this syndrome should avoid certain physical activities like swimming. Lifestyle modification follows the diagnosis, as well as avoidance of certain drugs which affect the QT interval, and the list is very long. At www.qtdrugs.org, you can find a long list of agents which should be avoided. ${ }^{14}$

The same holds true for patients with Brugada syndrome, where, following diagnosis, it is important to avoid fever and to treat any fever that does occur early. Certain drugs should also be avoided in patients with Brugada syndrome, a list of which can be found online at www. brugadadrugs.org. ${ }^{15}$

The ECG is a valuable tool for detecting people at risk of SCD, but requires further supporting investigation, such as taking a family history. In those who are found to be at risk, we should prescribe certain drugs, such as hydroquinidine in patients with Brugada syndrome, or appropriate medical intervention in patients with short QT syndrome. Implantable cardioverter defibrillator (ICD) implantation is a very effective measure to prevent SCD in high-risk patients.

Recently, it has been shown that catheter ablation is feasible in patients with Brugada syndrome, especially in those who have recurrent ventricular fibrillation episodes. ${ }^{16}$ Further research will need to be done where a preventive ablation will potentially cure patients with Brugada syndrome by ablating the substrate in the right ventricular outflow tract, either from the endocardium or epicardium.

In high-risk patients, identified by the ECG - and the ECG is only a screening tool - further diagnostic workup, including family history and cardiac imaging using magnetic resonance imaging, is required. Patients can then be further risk-stratified by a diagnostic electrophysiological study. In high-risk patients, ICD implant is warranted.

The European guidelines on the management and prevention of SCD in high-risk patients, for example those with hypertrophic cardiomyopathy, agreed that patients with a 5-year mortality of dying suddenly $>5 \%$, should receive an ICD. It has been shown by several groups that treating those people at high risk by ICD implantation can reduce risk of SCD. ${ }^{17,18}$ ]
1. Martens E, Sinner MF, Siebermair J, et al. Incidence of sudden cardiac death in Germany: results from an emergency medical service registry in Lower Saxony. Europace. 2014;16:1752-8.

2. de Vreede-Swagemakers IJ, Gorgels AP, Dubois-Arbouw WI, et al. Out-of-hospital cardiac arrest in the 1990's: a population-based study in the Maastricht area on incidence, characteristics and survival. J Am Coll Cardiol. 1997;30:1500-5.

3. Shen $\mathrm{L}$, Jhund $\mathrm{PS}$, Petrie MC et al. Declining risk of sudden death in heart failure. N Eng/ Med. 2017;377:41-51.

Koester MC. A review of sudden cardiac death in young athletes and strategies for preparticipation cardiovascular screening. J Athl Train. 2001;36:197-204.

5. Priori SG, Schwartz PJ, Napolitano C, et al. Risk stratification in the long-QT syndrome. N Eng/ J Med. 2003;348:1866-74.

Jouven X, Desnos M, Guerot C, Ducimetière P. Predicting sudden death in the population: the Paris Prospective Study I. sudden death in the population:
Circulation. 1999:99:1978-83.

7. Aro AL, Reinier K, Rusinaru C, et al. Electrical risk score beyond the left ventricular ejection fraction: prediction of sudden cardiac death in the Oregon Sudden Unexpected Death Study and the Atherosclerosis Risk in Communities Study. Eur Heart J. 2017:38:3017-25.

8. Rautaharju PM, Zhang ZM, Warren J, et al. Electrocardiographic predictors of coronary heart disease and sudden cardiac deaths in men and women free from cardiovascular disease in the Atherosclerosis Risk in Communities study. J Am Heart Assoc. 2013;2:e000061

9. Giustetto C, Schimpf R, Mazzanti A, et al. Long-term follow-up of patients with short QT syndrome. J Am Coll Cardiol. 2011;58:587-95.

10. Giustetto C, Di Monte F, Wolpert C, et al. Short QT syndrome: clinical findings and diagnostic-therapeutic implications. Eur Heart J. 2006:27:2440-7.

11. Brugada P, Brugada J. Right bundle branch block, persistent ST segment elevation and sudden cardiac death: a distinct clinica and electrocardiographic syndrome. A multicenter report. and electrocardiographic syndrom

12. Haïssaguerre M, Derval N, Sacher F, et al. Sudden cardiac arrest associated with early repolarization. N Eng/ I Med. 2008;358:2016-23.
13. Corrado D, Basso C, Pavei A et al. Trends in sudden cardiovascular death in young competitive athletes after implementation of a preparticipation screening program JAMA. 2006;296:1593-601.

14. CredibleMeds ${ }^{\oplus}$. Congenital LQTS and drugs to avoid list. Available at: https://crediblemeds.org/everyone/congenitallong-qt-and-drugs-avoid/ (accessed 11 December 2018).

15. Brugada Foundation. Drugs to be avoided by Brugada syndrome patients. Available at: www.brugadadrugs.org/avoid/ (accessed 11 December 2018)

16. Shelke A, Tachil A, Saggu D et al. Catheter ablation for electrical storm in Brugada syndrome: Results of substrate based ablation. Indian Heart J. 2018;70:296-302.

17. Stella BM, Alessandro Z. ICD implantation practice within Europe: how to explain the differences beyond economy? J Atr Fibrillation. 2015:8:1262.

18. Varr BC, Zarafshar S, Coakley T et al. Implantable cardioverterdefibrillator placement in patients with cardiac amyloidosis. Heart Rhythm. 2014;11:158-62. 